\title{
Effects of Slip Factors and Couple Stresses for Stagnation Point Flow over a Moving Plate
}

\author{
Najeeb Alam Khan and Fatima Riaz \\ Department of Mathematical Sciences, University of Karachi, Karachi 75270, Pakistan \\ Correspondence should be addressed to Najeeb Alam Khan; njbalam@yahoo.com
}

Received 25 July 2013; Accepted 11 September 2013

Academic Editors: E. V. Rebrov and D. Sun

Copyright ( 2013 N. A. Khan and F. Riaz. This is an open access article distributed under the Creative Commons Attribution License, which permits unrestricted use, distribution, and reproduction in any medium, provided the original work is properly cited.

\begin{abstract}
An investigation is presented for the two-dimensional and axisymmetric stagnation flows of a couple stress fluids intrude on a moving plate under partial slip conditions. The governing partial differential equations are converted into ordinary differential equations by a similarity transformation. The important physical parameters of skin friction coefficients of the fluid are also obtained. The homotopy analysis method (HAM) is employed to obtain the analytical solution of the problem. Also, the convergence of the solutions is established by plotting graphs of convergence control parameter. The impacts of couple stresses and slip conditions on the flow and temperature of the fluid have been observed. The numerical comparison for the considered fluid is compared with previous solutions as special case.
\end{abstract}

\section{Introduction}

The fluids exhibiting a boundary slip are important in industrial applications, for example, the polishing of artificial heart valves, rarefied fluid problems, and flow on multiple interfaces. There are many cases where no slip condition is replaced with Navier's partial slip condition. Partial slip condition on solid boundary occurs in many problems such as oscillatory flow channel, transient flow, some coated surfaces, some rough or porous surfaces, and heat transfer on moving plate. The flow on a moving plate is termed as a basic content for convection processes. The partial slip condition on a moving plate was considered by Wang [1]; the steady, laminar, axis-symmetric flow of a Newtonian fluid due to a stretching sheet with partial slip was studied by Ariel [2], Nadeem et al. [3] investigated steady state rotating and MHD flow of a third grade fluid past a rigid plate with slip; flow and heat transfer of a non-Newtonian fluid past a stretching sheet with partial slip are considered by Sahoo [4], and Jamil and Khan [5] considered the slip effects on fractional viscoelastic fluids; the steady boundary layer flow past a moving horizontal flat plate with a slip effect is studied by Kumaran and Pop [6].
The theory of couple stresses, introduced by Stokes [7], explain the rheological behavior of various complex nonNewtonian fluids with body stresses and body couples which cannot be illustrated by the classical theory of continuum mechanics. Due to the rotational interaction of particles, the force-stress tensor is not symmetric and flow behaviors of such fluids are not similar to the Newtonian ones. It draws the researcher's attention with the growing applications of such fluids in engineering, biomedical, and chemical industries. The peristaltic transport of a couple stress fluid in an asymmetric channel with the effect of the induced magnetic field has been considered by Nadeem and Akram [8], Khan et al. [9] investigate the approximate solution of couple stress fluid with expanding or contracting porous channel, Ramana Murthy and Nagaraju considered the flow of a couple stress fluid generated by a circular cylinder subjected to longitudinal and torsional oscillations [10], Hayat and colleagues observed that the velocity and the boundary layer thickness are decreasing functions of the couple stress fluid parameter in his study of melting heat transfer in the boundary layer flow of a couple stress fluid over a stretching surface [11], and the time dependence of the run up flow of a couple stress fluid between rigid parallel plates is examined 
by Devakar and Iyengar [12] in which the flow is induced by a constant pressure gradient which is suddenly withdrawn and the parallel plates are set to move instantaneously with different velocities in the direction of the applied pressure gradient.

In present paper, the effect of velocity slip factor $\lambda$ and thermal slip factor $\beta$ in two- dimensional and axisymmetric stagnation flows of a couple stress fluid are considered. Heat transfer analysis has also been taken into account. The problem is solved and analyzed with the application of HAM. The HAM provides a simple way to ensure convergence of the solution. It does not only optimize the solution but also gives freedom to choose the base function. The homotopy analysis method contains the auxiliary parameter $\hbar$, which provides a straightforward way to adjust and control the convergence region of the series solution. The HAM can overcome the restrictions and limitations of perturbation methods $[13,14]$. It has been successfully applied to many types of nonlinear problems such as the nonlinear equations arising in heat transfer [15], the nonhomogeneous Blasius problem [16], the generalized three-dimensional MHD flow over a porous stretching sheet [17], the axisymmetric flow, and heat transfer of a second grade fluid past a stretching sheet [18], and Kawahara equation [19], and an analytical study for the effects of slip factors on unsteady stagnation point flow and heat transfer towards a stretching sheet has also been carried out through HAM [20]. The solution can be expressed with the help of different linear operators, out of which some converge to the exact solution faster than others.

\section{Formulation of the Problem}

Let us consider a two-dimensional stagnation flow with heat transfer in the $x-z$ plane moving with velocity $U$ in the $x$-direction and velocity $V$ in $y$-direction (as shown in Figure 1). The partial slip condition on the surface of moving plate for both motion of the fluid and heat transfer is also considered. Under the considered assumptions, in the absence of body forces and body couples, the momentum and energy equations of an incompressible couple stress fluid are presented by the following equations:

$$
\begin{gathered}
\nabla \cdot \mathbf{V}=0 \\
\rho\left(\frac{\partial \mathbf{V}}{\partial t}+(\mathbf{V} \cdot \nabla) \mathbf{V}\right)=-\nabla p+\mu \nabla^{2} \mathbf{V}-\gamma \nabla^{4} \mathbf{V} \\
\rho C_{p}\left(\frac{\partial T}{\partial t}+(\mathbf{V} \cdot \nabla) T\right)=k \nabla^{2} T .
\end{gathered}
$$

2.1. The Two-Dimensional Flow. The potential flow far from the plate is given by

$$
\begin{gathered}
u=a x, \quad v=0, \quad w=-a z, \\
p=p_{0}-\rho a^{2} \frac{\left(x^{2}+z^{2}\right)}{2},
\end{gathered}
$$

where, $u, v, w$ are velocity components in the Cartesian $x, y, z$ directions, $a$ is the strength of the stagnation flow, $\rho$ is the density, $p$ is the pressure, and $p_{0}$ is the stagnation pressure.

For couple stress flow, we set

$$
\begin{gathered}
u=a x f^{\prime}(\eta)+U g(\eta), \quad v=V h(\eta), \\
w=-\sqrt{a} \nu f(\eta), \\
\theta(\eta)=\frac{T-T_{\infty}}{T_{0}-T_{\infty}}, \quad \eta \equiv \sqrt{\frac{a}{v}} z, \\
p=p_{0}-\rho\left(\frac{a^{2} x^{2}}{2}+\frac{w^{2}}{2}-v w_{z}+\gamma w_{z, z, z}\right),
\end{gathered}
$$

where $\gamma$ is the couple stress fluid parameter and $v$ is the kinematic viscosity. The subscript $z$ represents differentiation with respect to $z$. Using (3)-(4), the momentum and energy equations are reduced to ordinary differential equations

$$
\begin{gathered}
f^{\prime \prime \prime}+f f^{\prime \prime}-\left(f^{\prime}\right)^{2}-\xi f^{(5)}+1=0, \\
g^{\prime \prime}+f g^{\prime}-f^{\prime} g-\xi g^{(4)}=0, \\
h^{\prime \prime}+f h^{\prime}-\xi h^{(4)}=0 \\
\theta^{\prime \prime}+\operatorname{Pr} f \theta^{\prime}=0
\end{gathered}
$$

where $\operatorname{Pr}=\nu / \alpha$ is Prandtl number and $\xi=a \gamma /\left(\nu^{2} \rho\right)$ is the couple stress parameter.

On the plate, velocity slip and thermal slip similar to Navier's condition is

$$
\begin{gathered}
u-U=N\left(\rho v u_{z}-\gamma u_{z z z}\right), \\
v-V=N\left(\rho \nu v_{z}-\gamma v_{z z z}\right), \quad T-T_{0}=S T_{z},
\end{gathered}
$$

where $N$ and $S$ are slip constants. Substitution of (3) yields

$$
\begin{gathered}
f^{\prime}(0)=\lambda\left(f^{\prime \prime}(0)-\xi f^{\prime \prime \prime \prime}(0)\right), \\
g(0)=1+\lambda\left(g^{\prime}(0)-\xi g^{\prime \prime \prime}(0)\right), \\
h(0)=1+\lambda\left(h^{\prime}(0)-\xi h^{\prime \prime \prime}(0)\right), \\
\theta(0)=1+\beta \theta^{\prime}(0),
\end{gathered}
$$

where $\lambda=N \rho \sqrt{\nu a}$ is the nondimensional slip factor and $\beta=$ $S \sqrt{a / v}$ is the thermal slip factor. For an impermeable plate and a potential flow which is recovered at infinity, we have

$$
\begin{gathered}
f(0)=0, \quad f^{\prime}(\infty)=1, \quad g(\infty)=0, \\
h(\infty)=0, \quad \theta(\infty)=0 .
\end{gathered}
$$

2.2. The Axisymmetric Stagnation Point Flow. The stagnation flow in the $x-z$ plane is moving two-dimensionally with 


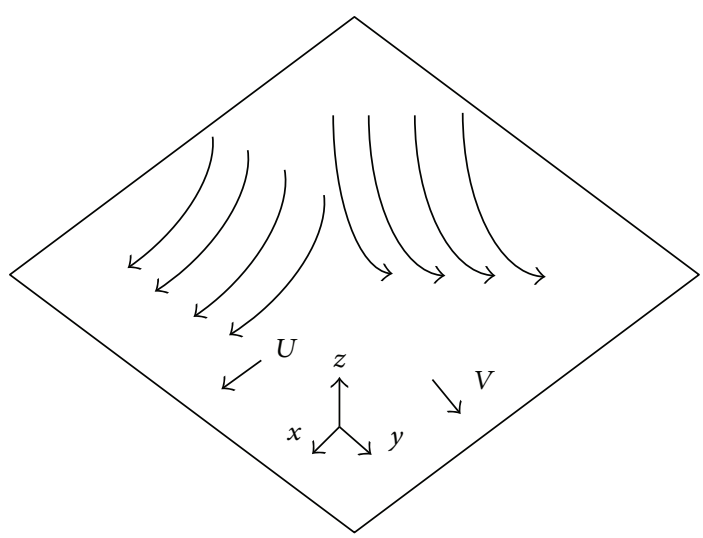

(a)

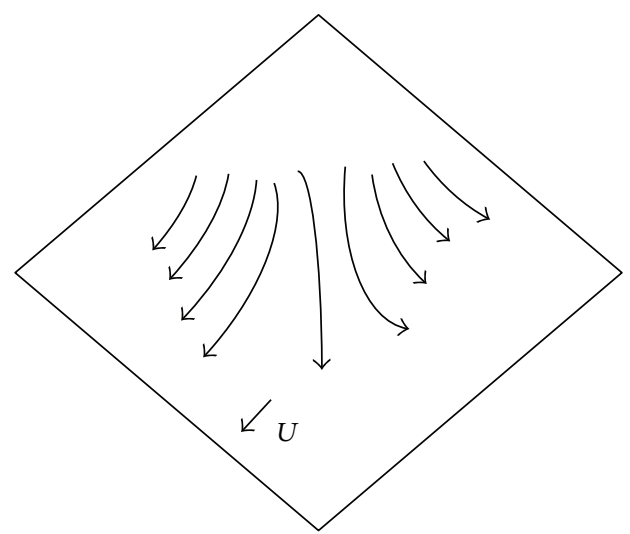

(b)

FIgUre 1: (a) Two-dimensional stagnation flow. (b) Axisymmetric stagnation flow.

velocity $U$ in the $x$ direction and velocity $V$ in the $y$ direction. The potential flow far from the plate is given by

$$
\begin{gathered}
u=a x, \quad v=a y, \quad w=-2 a z, \\
p=p_{0}-\rho\left[\frac{\left(x^{2}+z^{2}\right)}{2}+2 a^{2} z^{2}\right] .
\end{gathered}
$$

We introduce the following transformations:

$$
\begin{gathered}
u=a x f^{\prime}(\eta)+U g(\eta), \quad v=a y f^{\prime}(\eta), \\
w=-2 \sqrt{a v} f(\eta), \\
p=p_{0}-\rho\left[\frac{a^{2}\left(x^{2}+y^{2}\right)}{2}+\frac{w^{2}}{2}+\nu w_{z}+\gamma w_{z, z, z}\right] .
\end{gathered}
$$

The equations of motion and energy are reduced to ordinary differential equations:

$$
\begin{gathered}
f^{\prime \prime \prime}+2 f f^{\prime \prime}-\left(f^{\prime}\right)^{2}-\xi f^{(5)}+1=0, \\
g^{\prime \prime}+2 f g^{\prime}-f^{\prime} g-\xi g^{(4)}=0, \\
\theta^{\prime \prime}+2 \operatorname{Pr} f \theta^{\prime}=0
\end{gathered}
$$

with the same boundary conditions as in (7)-(8).

The shear stress of the fluid for two-dimensional and axisymmetric flows can be found as

$$
\begin{aligned}
& \tau_{x z}=\left.\left(\mu\left(\frac{\partial u}{\partial z}\right)-\xi\left(\frac{\partial^{3} u}{\partial z^{3}}\right)\right)\right|_{z=0}, \\
& \tau_{y z}=\left.\left(\mu\left(\frac{\partial v}{\partial z}\right)-\xi\left(\frac{\partial^{3} v}{\partial z^{3}}\right)\right)\right|_{z=0} .
\end{aligned}
$$

Hence, the local skin friction coefficients are given by

$$
\begin{aligned}
\operatorname{Re}_{x}^{1 / 2} C_{f} & =f^{\prime \prime}(0)-\xi f^{\prime \prime \prime \prime}(0), \\
C_{g} & =\frac{g^{\prime}(0)-\xi g^{\prime \prime \prime}(0)}{\sqrt{U^{2} / a \nu}}, \\
C_{g} & =\frac{h^{\prime}(0)-\xi h^{\prime \prime \prime}(0)}{\sqrt{V^{2} / a \nu}},
\end{aligned}
$$

where $\operatorname{Re}_{x}^{1 / 2}=\sqrt{a x^{2} / \nu}$ is the local Reynolds numbers.

\section{Solution of the Problem}

For the two-dimensional problem presented in (5), the course of action for the HAM solution, we select

$$
\begin{gathered}
f_{0}=\frac{-1}{1+\lambda}+\eta+\frac{e^{-\eta}}{1+\lambda}, \quad g_{0}=\frac{e^{-\eta}}{1+\lambda}, \\
h_{0}=\frac{e^{-\eta}}{1+\lambda}, \quad \theta_{0}=\frac{e^{-\eta}}{1+\beta}
\end{gathered}
$$

as the initial approximation of $f_{0}, g_{0}, h_{0}$, and $\theta_{0}$, respectively, which satisfy the following linear operator and corresponding boundary conditions:

$$
\mathscr{L}_{1}=\frac{d^{3}}{d \eta^{3}}+\frac{d^{2}}{d \eta^{2}}, \quad \mathscr{L}_{2}=\frac{d^{2}}{d \eta^{2}}-1
$$

such that

$$
\mathscr{L}_{1}\left[c_{1}+c_{2} e^{\eta}+c_{3} e^{-\eta}\right]=0, \quad \mathscr{L}_{2}\left[c_{4} e^{\eta}+c_{5} e^{-\eta}\right]=0,
$$

where $c_{1}, c_{2}, \ldots, c_{5}$ are arbitrary constants. 
The following nonlinear operators are defined as

$$
\begin{aligned}
& N_{1}[\tilde{f}(\eta, p)]=\frac{\partial^{3} \tilde{f}(\eta, p)}{\partial \eta^{3}}+\tilde{f}(\eta, p) \frac{\partial^{2} \tilde{f}(\eta, p)}{\partial \eta^{2}} \\
& -\left(\frac{\partial \tilde{f}(\eta, p)}{\partial \eta}\right)^{2}-\xi \frac{\partial^{5} \tilde{f}(\eta, p)}{\partial \eta^{5}}+1, \\
& N_{2}[\tilde{f}(\eta, p), \tilde{g}(\eta, p)]=\frac{\partial^{2} \tilde{g}(\eta, p)}{\partial \eta^{2}}+\tilde{f}(\eta, p) \frac{\partial \widetilde{g}(\eta, p)}{\partial \eta} \\
& -\frac{\partial \tilde{f}(\eta, p)}{\partial \eta} \widetilde{g}(\eta, p)-\xi \frac{\partial^{4} \tilde{g}(\eta, p)}{\partial \eta^{4}}, \\
& N_{3}[\tilde{f}(\eta, p), \tilde{h}(\eta, p)]=\frac{\partial^{2} \widetilde{h}(\eta, p)}{\partial \eta^{2}}+\tilde{f}(\eta, p) \frac{\partial \widetilde{h}(\eta, p)}{\partial \eta} \\
& -\xi \frac{\partial^{4} \widetilde{h}(\eta, p)}{\partial \eta^{4}} \\
& N_{4}[\tilde{f}(\eta, p), \tilde{\theta}(\eta, p)]=\frac{\partial^{2} \widetilde{\theta}(\eta, p)}{\partial \eta^{2}}+\operatorname{Pr} \tilde{f}(\eta, p) \frac{\partial \tilde{\theta}(\eta, p)}{\partial \eta}
\end{aligned}
$$

and then construct the zeroth-order deformation equations:

$$
\begin{aligned}
& (1-p) \mathscr{L}_{1}\left[\tilde{f}(\eta, p)-f_{0}(\eta)\right] \\
& -p \hbar H_{1}(\eta) N_{1}[\tilde{f}(\eta, p)]=0, \\
& (1-p) \mathscr{L}_{2}\left[\widetilde{g}(\eta, p)-g_{0}\right] \\
& -p \hbar H_{2}(\eta) N_{2}[\tilde{f}(\eta, p), \widetilde{g}(\eta, p)]=0, \\
& (1-p) \mathscr{L}_{2}\left[\widetilde{h}(\eta, p)-h_{0}\right] \\
& -p \hbar H_{3}(\eta) N_{3}[\tilde{f}(\eta, p), \tilde{h}(\eta, p)]=0, \\
& (1-p) \mathscr{L}_{2}\left[\tilde{\theta}(\eta, p)-\theta_{0}(\eta)\right] \\
& -p \hbar H_{4}(\eta) N_{4}[\tilde{f}(\eta, p), \tilde{\theta}(\eta, p)]=0, \\
& \tilde{f}^{\prime}(0, p)=\lambda\left(\tilde{f}^{\prime \prime}(0, p)-\xi \tilde{f}^{\prime \prime \prime \prime}(0, p)\right), \\
& \tilde{f}(0, p)=0, \\
& \widetilde{g}(0, p)=1+\lambda\left(\widetilde{g}^{\prime}(0, p)-\xi \widetilde{g}^{\prime \prime \prime}(0)\right), \\
& \widetilde{h}(0, p)=1+\lambda\left(\widetilde{h}^{\prime}(0, p)-\xi \widetilde{h}^{\prime \prime \prime}(0)\right), \\
& \tilde{\theta}(0, p)=1+\beta \widetilde{\theta}^{\prime}(0, p), \\
& \tilde{f}^{\prime}(\infty, p)=1, \quad \tilde{g}(\infty, p)=0, \quad \tilde{h}(\infty, p)=0, \\
& \tilde{\theta}(\infty, p)=0
\end{aligned}
$$

in which $p \in[0,1]$ is the embedding parameter and $\hbar$ is the auxiliary nonzero parameter and $H_{i}(\eta), i=1,2,3,4$ are the auxiliary functions. Here, we selected the auxiliary functions as $H_{k}=e^{-\eta}, k=1,2,3,4$.

By Taylor's theorem,

$$
\begin{aligned}
& f(\eta, p)=f_{0}(\eta)+\sum_{m=1}^{\infty} f_{m}(\eta) p^{m}, \\
& g(\eta, p)=g_{0}(\eta)+\sum_{m=1}^{\infty} g_{m}(\eta) p^{m}, \\
& h(\eta, p)=h_{0}(\eta)+\sum_{m=1}^{\infty} h_{m}(\eta) p^{m}, \\
& \theta(\eta, p)=\theta_{0}(\eta)+\sum_{m=1}^{\infty} \theta_{m}(\eta) p^{m} .
\end{aligned}
$$

The $m$ th-order deformation problems with the corresponding boundary conditions are given by

$$
\begin{gathered}
\mathscr{L}_{1}\left[f_{m}-\chi_{m} f_{m-1}\right]=\hbar e^{-\eta} R_{1}\left[f_{m-1}\right], \\
\mathscr{L}_{2}\left[g_{m}-\chi_{m} g_{m-1}\right] \hbar e^{-\eta} R_{2}\left[f_{m-1}, g_{m-1}\right], \\
\mathscr{L}_{2}\left[h_{m}-\chi_{m} h_{m-1}\right]=\hbar e^{-\eta} R_{3}\left[f_{m-1}, h_{m-1}\right], \\
\mathscr{L}_{2}\left[\theta_{m}-\chi_{m} \theta_{m-1}\right]=\hbar e^{-\eta} R_{4}\left[f_{m-1}, \theta_{m-1}\right], \\
f_{m}^{\prime}(0)=\lambda\left(f_{m}^{\prime \prime}(0)-\xi f^{\prime \prime \prime \prime}(0)\right), \quad f_{m}(0)=0, \\
g_{m}(0)=\lambda\left(g_{m}^{\prime}(0)-\xi g^{\prime \prime \prime}(0)\right), \\
h_{m}(0)=\lambda\left(h_{m}^{\prime}(0)-\xi h^{\prime \prime \prime}(0)\right), \\
\theta_{m}(0)=\beta \theta_{m}^{\prime}(0), \quad f_{m}^{\prime}(\infty)=0, \\
g_{m}(\infty)=0, \quad h_{m}(\infty)=0, \\
\theta_{m}(\infty)=0,
\end{gathered}
$$

where

$$
\begin{gathered}
R_{1}\left[f_{m-1}\right]=f_{m-1}^{\prime \prime \prime}+\sum_{i=0}^{m-1} f_{i} f_{m-1-i}^{\prime \prime}-\sum_{i=0}^{m-1} f_{i}^{\prime} f_{m-1-i}^{\prime} \\
-\xi f_{m-1}^{(5)}+\left(1-\chi_{m}\right), \\
R_{2}\left[f_{m-1}, g_{m-1}\right]=g_{m-1}^{\prime \prime}+\sum_{i=0}^{m-1} f_{i} g_{m-1-i}^{\prime} \\
-\sum_{i=0}^{m-1} f_{i}^{\prime} g_{m-1-i}-\xi g_{m-1}^{(4)}, \\
R_{3}\left[f_{m-1}, h_{m-1}\right]=h_{m-1}^{\prime \prime}+\sum_{i=0}^{m-1} f_{i} h_{m-1-i}^{\prime}-\xi h_{m-1}^{(4)}, \\
R_{4}\left[f_{m-1}, \theta_{m-1}\right]=\theta_{m-1}^{\prime \prime}+\operatorname{Pr} \sum_{i=0}^{m-1} f_{i} \theta_{m-1-i}^{\prime}, \\
\chi_{m}= \begin{cases}0, & m \leq 1, \\
1, & m \geq 2 .\end{cases}
\end{gathered}
$$




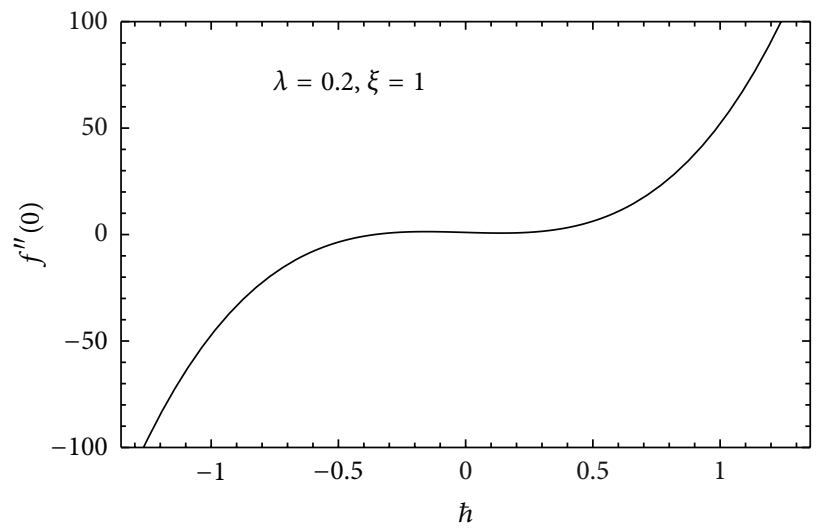

(a)

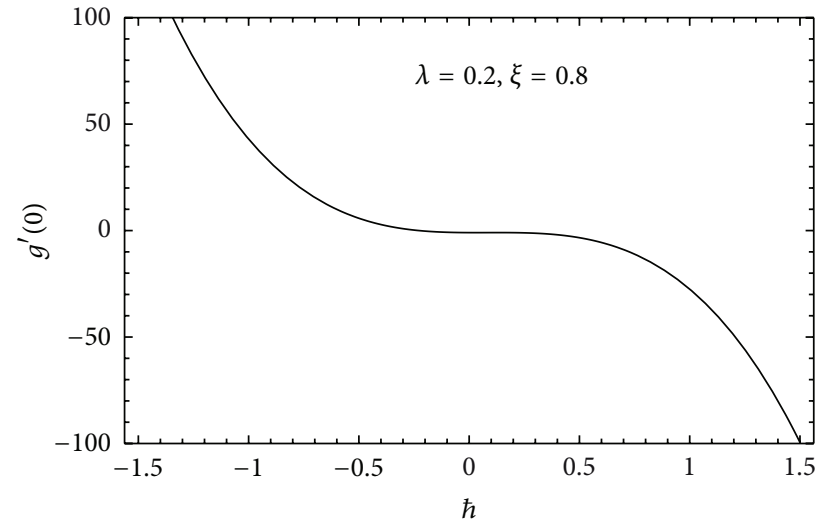

(b)

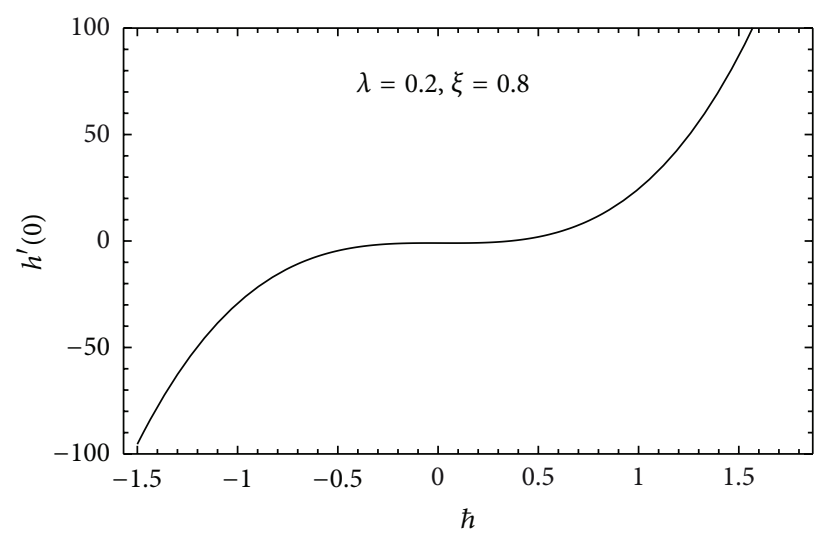

(c)

FIGURE 2: The $\hbar$ curves for 6th order of approximation of HAM when $\lambda=0.2, \xi=1$. (a) for $f^{\prime \prime}(0)$, (b) for $g^{\prime}(0)$, (c) for $h^{\prime}(0)$.

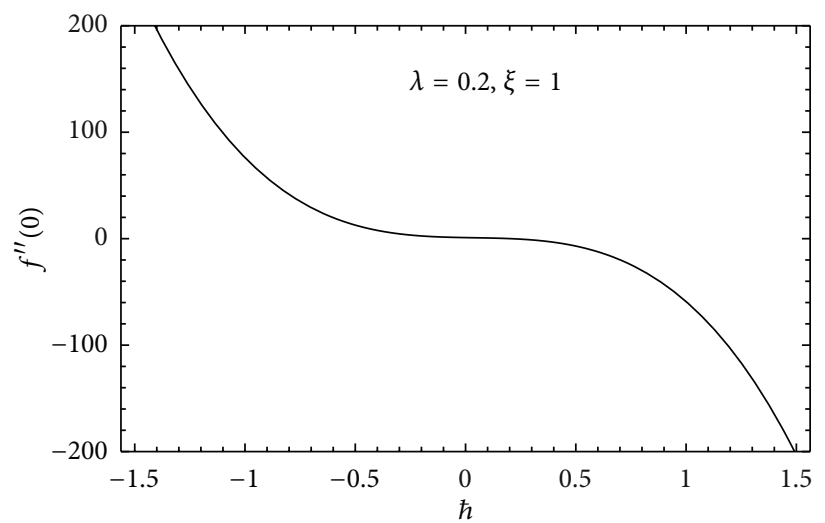

(a)

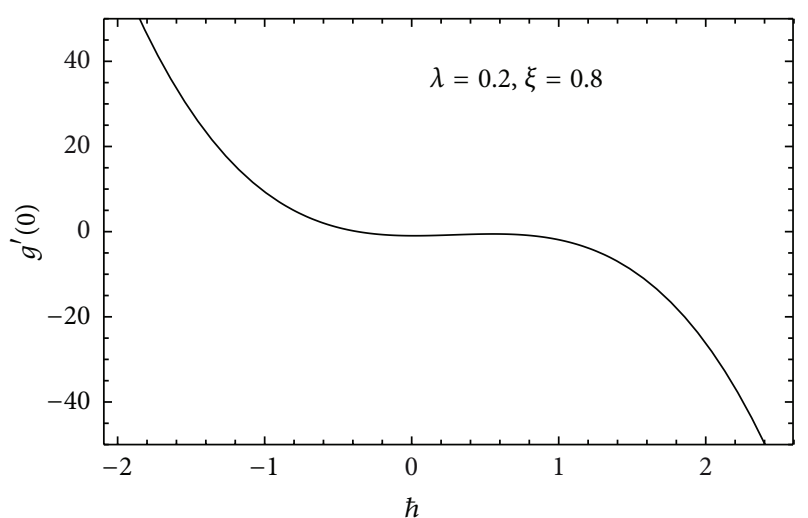

(b)

Figure 3: The $\hbar$ curves for 6 th order of approximation of HAM when $\lambda=0.2, \xi=1$. (a) for $f^{\prime \prime}(0)$, (b) for $g^{\prime}(0)$.

According to the procedure defined previously, it is easy to solve the linear equations (20), one after the other in the order $m$, especially by means of any computation software, such as Mathematica.

In a similar manner the solution of axisymmetric problem defined in (11) can also be determined by taking the same linear operators and initial guesses and proceeding in the same way as for two-dimensional problem.

\section{Results and Discussions}

The paper presents an analysis of the flow and heat transfer of the stagnation slip flow of a couple stress fluid over a moving plate. Approximate analytical solutions are derived for momentum and energy equations. We found analytic solutions corresponding to the heat transfer of stagnation slip flow over a moving plate by using symbolic software 


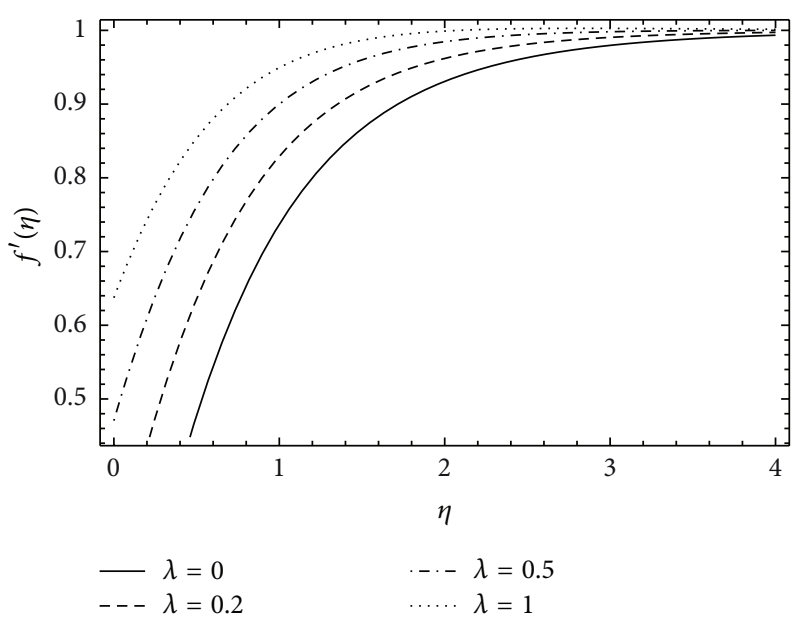

(a)

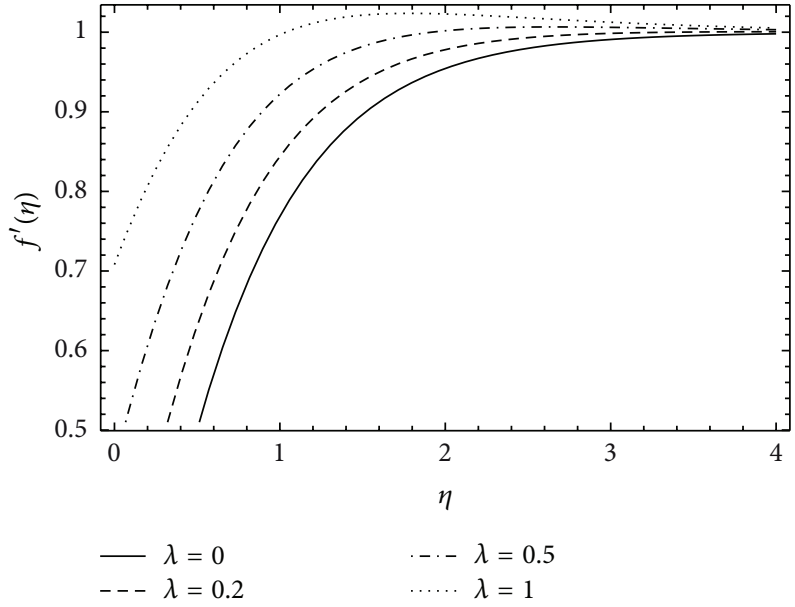

(b)

FIGURE 4: Effect of slip factor $\lambda$ on $f^{\prime}(\eta)$ (slip and no-slip cases) when $\hbar=-0.3, \xi=0.5$. (a) for two-dimensional and (b) for axisymmetric case.

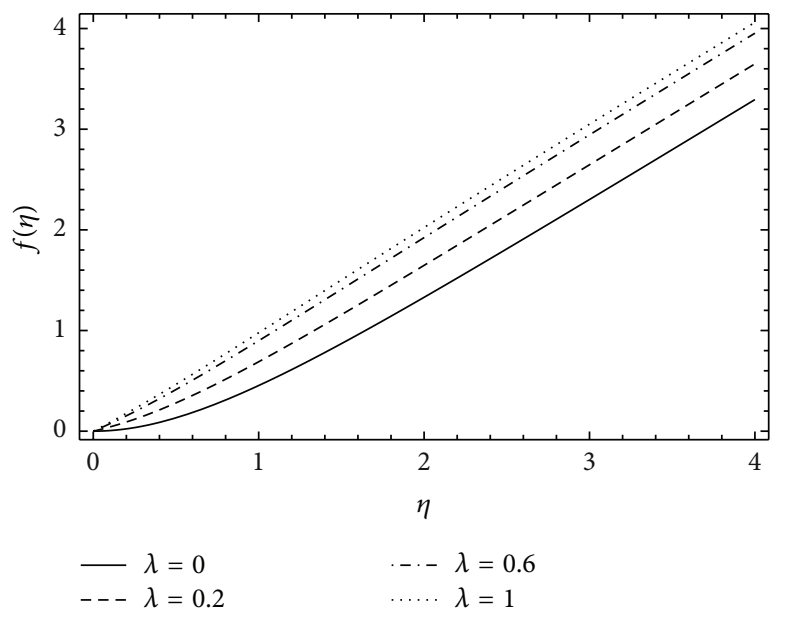

(a)

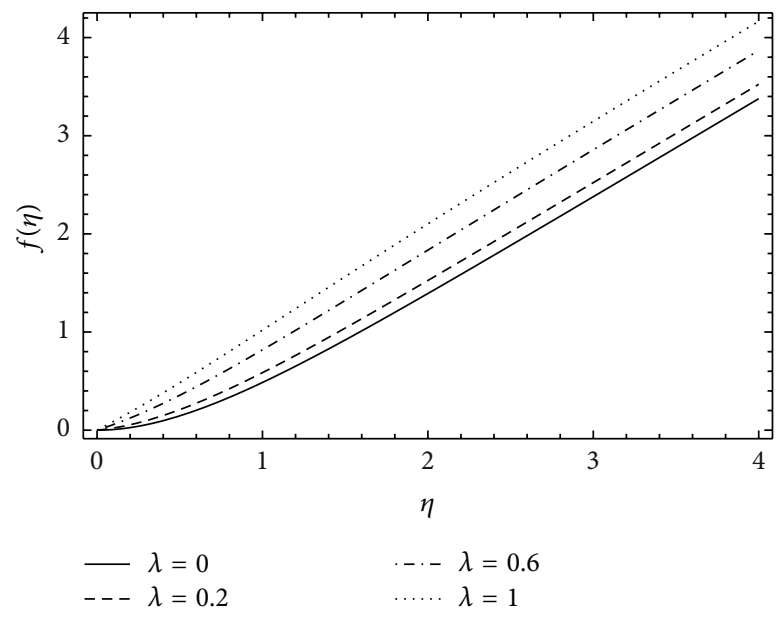

(b)

Figure 5: Effect of slip factor $\lambda$ on $f(\eta)$ (slip and no-slip cases) when $\hbar=-0.2, \lambda=0.2$ (a) for two-dimensional, and (b) for axisymmetric case.

Mathematica 9. The HAM is adopted to achieve the analytic solution of the problem. In HAM, a deformation equation defines a continuous variation from an initial approximation to the solution through an embedding parameter. The convergence region of the obtained solution can be determined with the help of embedding parameter $\hbar$ which provides the straight line parallel to $x$-axis in which the resultant solution is convergent. For the two-dimensional flow, Figures 2(a)$2(c)$, the $\hbar$ curves are plotted for $f^{\prime \prime}(0), g^{\prime}(0)$ and $h^{\prime}(0)$, and for axisymmetric flow, Figures 3(a) and 3(b) are plotted for $f^{\prime \prime}(0)$ and $g^{\prime}(0)$ that presents the admissible values of $\hbar$ under which the solution is convergent.

For both two-dimensional and axisymmetric stagnation flows, the effect of couple stress parameter $\xi$ on temperature and velocity profiles, the effects of velocity slip factor $\lambda$ on velocity components, and the effect of thermal slip factor $\beta$ on temperature, which in turn depends on velocity, are presented in the form of graphical and tabulated results. In Tables 1, 2, and 3 (for two-dimensional) and Tables 4 and 5 (for axisymmetric), the initial values of $\sqrt{\operatorname{Re}} C_{f},\left(\sqrt{U^{2} / a v}\right) C_{g}$, and $\left(\sqrt{V^{2} / a v}\right) C_{n}$ are presented for the various values of $\xi$ and $\lambda$. At $\xi=0$ the problem recovers the Newtonian flow problem of Wang [1]. The results in the tables are in agreement with the results of Wang [1] (for $\xi=0$ ) and follow the same trend for increasing slip factor $\lambda$ when there are couple stresses in the fluid. Figures 4 and 5 represent the influence of velocity slip factor $\lambda$ on $f^{\prime}$ and $f$. An increase in the slip factor $\lambda$ decelerates the velocity $f^{\prime}$ (see Figure 4 ). The increase in the function $f$ is observed with the increasing in slip factor as shown in Figure 5. It can be noticed from Figure 6 that positive increase in the couple stress parameter $\xi$ has the negative impact on the velocity $f^{\prime}$ of the couple stress fluid; it influences more on axisymmetric flow as compared to two-dimensional flow, whereas couple stress parameter $\xi$ 
TABLE 1: Initial values of $\sqrt{\operatorname{Re}} C_{f}$ for the two-dimensional stagnation flow when $\hbar=-0.61$ (bracket values from [1]).

\begin{tabular}{lccccc}
\hline$\lambda \backslash \xi$ & 0 & 0.1 & 0.2 & 0.3 & 0.4 \\
\hline 0 & $1.23260(1.23259)$ & 1.43251 & 2.58260 & 5.25453 & 3.82149 \\
0.2 & $1.04385(1.04259)$ & 1.17052 & 1.94111 & 2.60405 & 7.15871 \\
0.5 & $0.82483(0.82148)$ & 0.90095 & 1.38118 & 1.60141 & 4.90074 \\
1 & $0.59637(0.59346)$ & 0.63885 & 0.90465 & 0.82142 & 2.97625 \\
2 & $0.37692(0.37589)$ & 0.39660 & 0.51389 & 0.12349 & 1.44177 \\
10 & $0.094047(0.094036)$ & 0.09559 & 0.10423 & 0.05608 & 0.14926 \\
20 & $0.048476(0.048472)$ & 0.04889 & 0.05126 & 0.02097 & 0.021125 \\
50 & $0.019753(0.019752)$ & 0.01982 & 0.02022 & & 0.0587 \\
\hline
\end{tabular}

TABLE 2: Initial values of $\sqrt{U^{2} / a \nu} C_{g}$ for the two dimensional stagnation flow when $\hbar=-0.35$ (bracket values from [1]).

\begin{tabular}{|c|c|c|c|c|c|}
\hline$\lambda \backslash \xi$ & 0 & 0.1 & 0.2 & 0.3 & 0.4 \\
\hline 0 & $-0.83406(-0.81130)$ & -0.98987 & -1.37103 & -2.07901 & -3.19268 \\
\hline 0.2 & $-0.75213(-0.77521)$ & -0.84363 & -1.09389 & -1.59464 & -2.43874 \\
\hline 0.5 & $-0.63674(-0.67196)$ & -0.68416 & -0.83462 & -1.16169 & -1.75519 \\
\hline 1 & $-0.49511(-0.52189)$ & -0.51574 & -0.59259 & -0.78238 & -1.15175 \\
\hline 2 & $-0.33602(-0.34911)$ & -0.34277 & -0.37504 & -0.45978 & -0.64203 \\
\hline 10 & $-0.091548(-0.09248)$ & -0.09184 & -0.09389 & -0.10003 & -0.11507 \\
\hline 20 & $-0.047812(-0.04806)$ & -0.04788 & -0.048423 & -0.05008 & -0.05418 \\
\hline 50 & $-0.019642(0.019685)$ & -0.01965 & -0.019744 & -0.02002 & -0.02070 \\
\hline
\end{tabular}

TABLE 3: Initial values of $\sqrt{V^{2} / a \nu} C_{h}$ for the two dimensional stagnation flow when $\hbar=-0.5$ (bracket values from [1]).

\begin{tabular}{lccccc}
\hline$\lambda \backslash \xi$ & 0 & 0.1 & 0.2 & 0.3 & 0.4 \\
\hline 0 & $-0.68635(-0.57047)$ & -0.68046 & -0.51044 & 0.02198 & -0.34999 \\
0.2 & $-0.62273(-0.55440)$ & -0.63793 & -0.59093 & -0.54172 & -0.31703 \\
0.5 & $-0.53707(-0.50283)$ & -0.56007 & -0.58301 & -0.561178 & -0.27375 \\
1 & $-0.43014(-0.41618)$ & -0.45097 & -0.49846 & -0.56149 \\
2 & $-0.30360(-0.29951)$ & -0.31649 & -0.35525 & -0.10908 & -0.54462 \\
10 & $-0.08882(-0.08871)$ & -0.09018 & -0.04896 & -0.05324 & -0.06313932 \\
20 & $-0.047054(-0.04703)$ & -0.04744 & -0.01985 & -0.020635 & -0.02252 \\
50 & $-0.01951(-0.01951)$ & -0.019580 & & & \\
\hline
\end{tabular}

TABLE 4: Initial values of $\sqrt{\operatorname{Re}} C_{f}$ for the axisymmetric flow when $\hbar=-0.59$ (bracket values from [1]).

\begin{tabular}{lcccrr}
\hline$\lambda \backslash \xi$ & 0 & 0.1 & 0.2 & 0.3 & 1.61316 \\
\hline 0 & $1.31166(1.31193)$ & 1.35335 & 1.50955 & 1.85177 & 1.22802 \\
0.2 & $1.11399(1.11097)$ & 1.17608 & 1.44234 & 1.82827 & 2.07303 \\
0.5 & $0.873726(0.86688)$ & 0.94076 & 0.94791 & 1.54311 & 1.03573 \\
1 & $0.622290(0.6173)$ & 0.67807 & 0.59476 & 0.18272 & 2.44917 \\
2 & $0.386430(0.38526)$ & 0.41918 & 0.11831 & 0.07543 & 0.33549 \\
10 & $0.094590(0.09459)$ & 0.09752 & 0.05557 & 0.02463 \\
20 & $0.048630(0.04862)$ & 0.04943 & 0.02100 & 0.12409 \\
50 & $0.019780(0.01977)$ & 0.01992 & & 0.03398 \\
\hline
\end{tabular}

decelerates the velocity function $f$ for both two-dimensional and axisymmetric flows (see Figure 7).

Figure 8 illustrates the influence of velocity slip factor $\lambda$ (for both two-dimensional and axisymmetric flows) on the velocity component $g$. An increase in the slip factor $\lambda$ results in an exponential decay of $g$; the effect of couple stress parameter $\xi$ on the velocity component $g$ reveals that it supports the two-dimensional flow and the axisymmetric flow in axial direction (see Figure 9). From Figure 10, it is noted that the influence of velocity slip factor $\lambda$ on the translational velocity $h$ is a reducing function of slip factor $\lambda$. The effect of couple stress parameter $\xi$ on the translational velocity $h$ is plotted in Figure 11; it is observed that it is also a decreasing function of couple stress parameter. 
TABLE 5: Initial values of $\sqrt{U^{2} / a \nu} C_{g}$ for the axisymmetric flow when $\hbar=-0.3$ (bracket values from [1]).

\begin{tabular}{|c|c|c|c|c|c|}
\hline$\lambda \backslash \xi$ & 0 & 0.1 & 0.2 & 0.3 & 0.4 \\
\hline 0 & $-0.87723(-0.93873)$ & -0.98615 & -1.17030 & -1.43528 & -1.77243 \\
\hline 0.2 & $-0.78757(-0.88766)$ & -0.86814 & -1.02163 & -1.26119 & -1.58740 \\
\hline 0.5 & $-0.66257(-0.74987)$ & -0.72114 & -0.84428 & -1.05073 & -1.35156 \\
\hline 1 & $-0.51106(-0.56453)$ & -0.55219 & -0.64428 & -0.80782 & -1.06257 \\
\hline 2 & $-0.343538(-0.36643)$ & -0.36980 & -0.42972 & -0.54027 & -0.72341 \\
\hline 10 & $-0.092122(-0.09356)$ & -0.09888 & -0.11326 & -0.13908 & -0.18304 \\
\hline 20 & $-0.047969(-0.04835)$ & -0.05146 & -0.05867 & -0.07123 & -0.09205 \\
\hline 50 & $-0.019669(-0.01973)$ & -0.02109 & -0.02396 & -0.02883 & -0.03662 \\
\hline
\end{tabular}

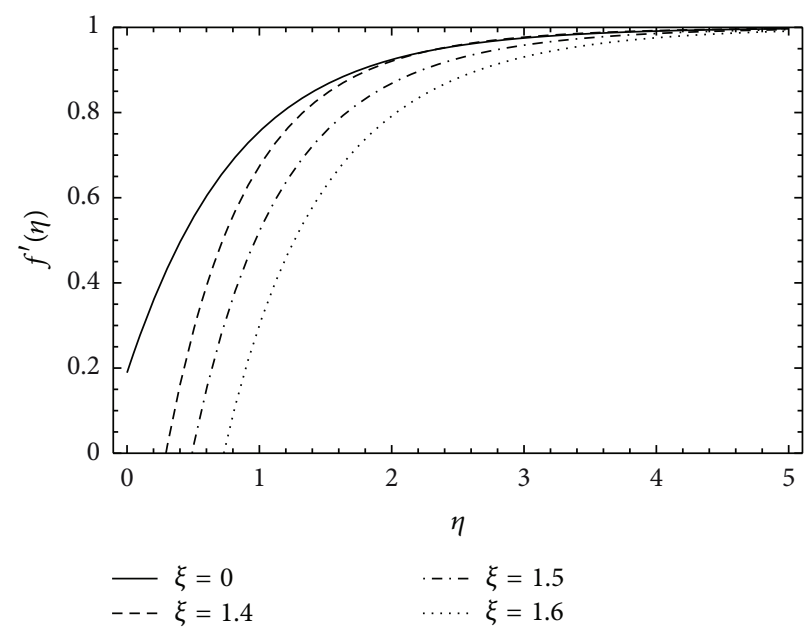

(a)

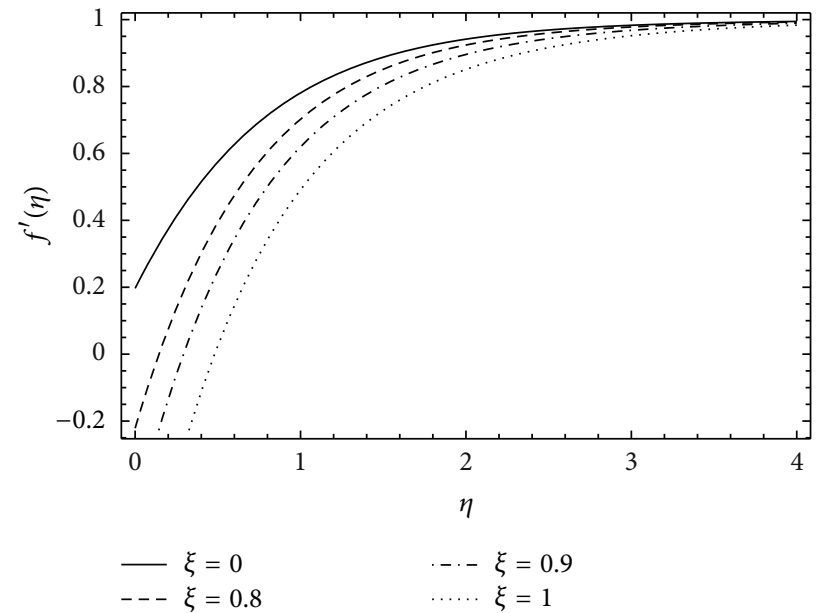

(b)

Figure 6: Effect of couple stress parameter $\xi$ on $f^{\prime}(\eta)$ (Newtonian and couple stress cases) when $\hbar=-0.2, \lambda=0.2$. (a) for two-dimensional and (b) for axisymmetric cases.

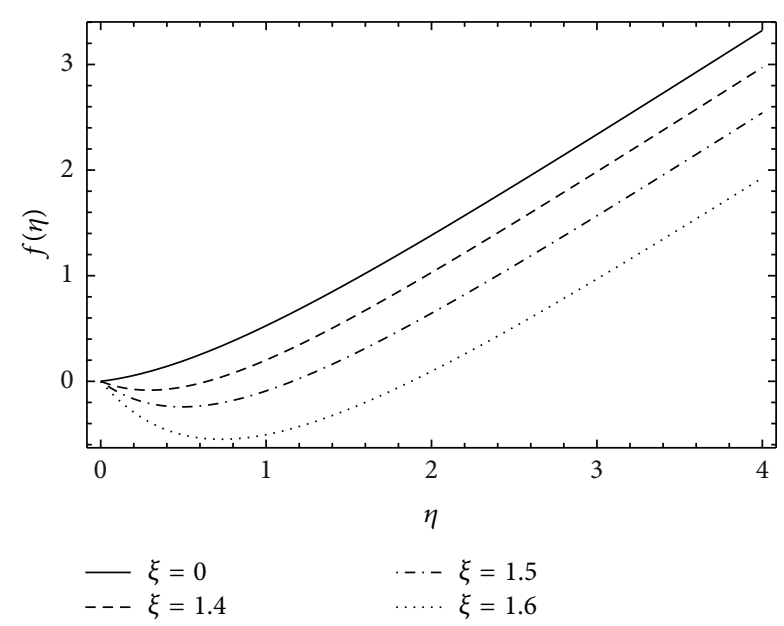

(a)

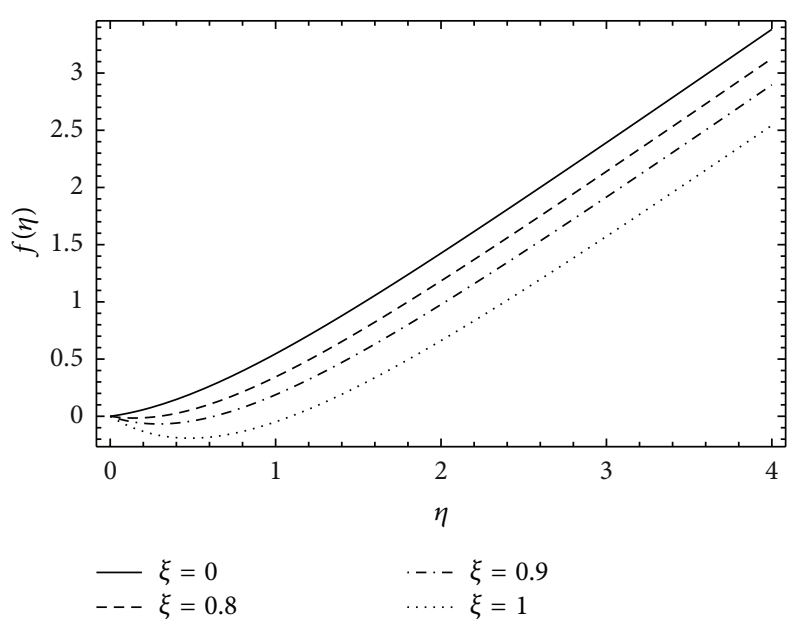

(b)

Figure 7: Effect of couple stress parameter $\xi$ on $f(\eta)$ (Newtonian and couple stress cases) when $\hbar=-0.2, \lambda=0.2$. (a) for two-dimensional and (b) for axisymmetric. 


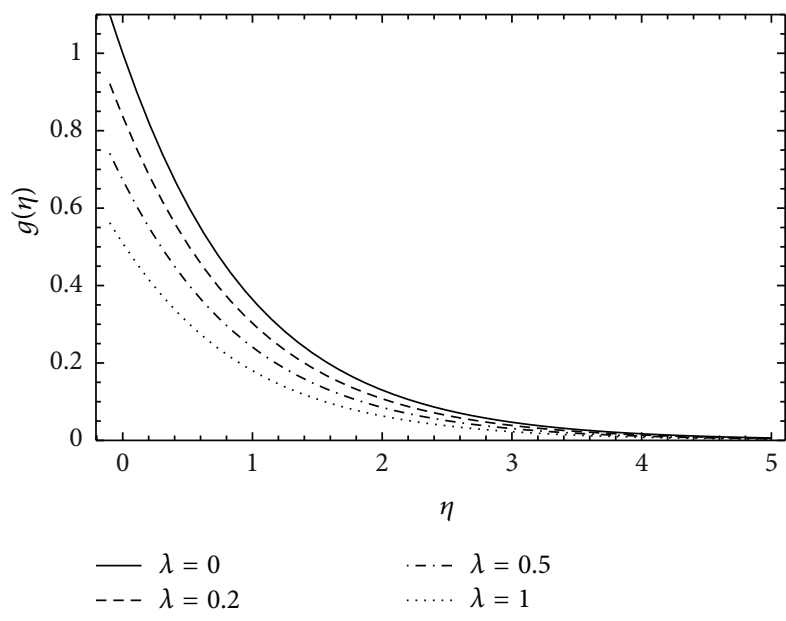

(a)

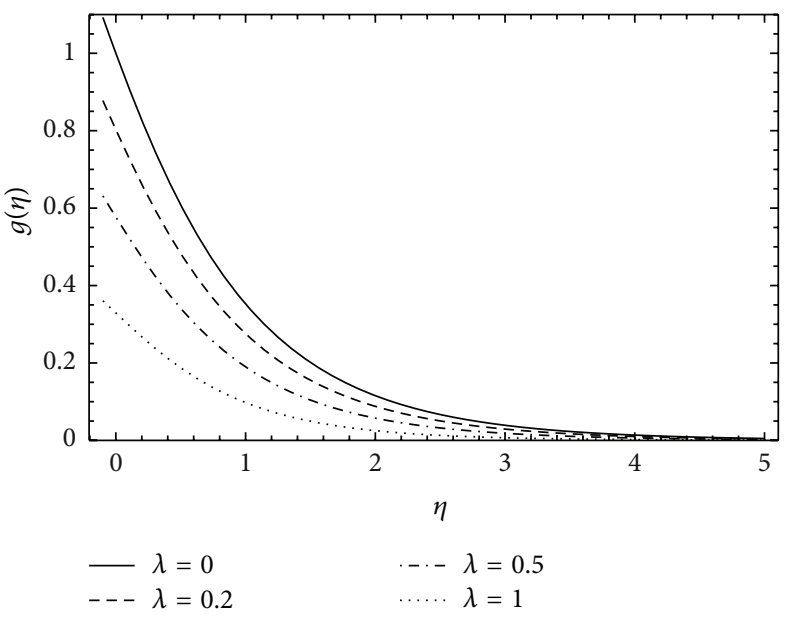

(b)

FIGURE 8: Effect of slip factor $\lambda$ on $g(\eta)$ (slip and no-slip cases) when $\hbar=-0.2, \xi=0.5$. (a) for two-dimensional flow and (b) for axisymmetric flow.

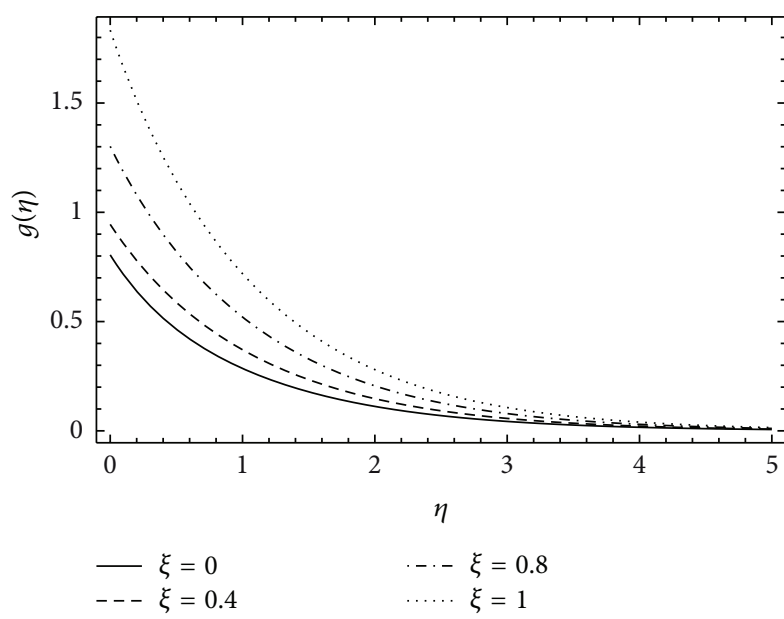

(a)

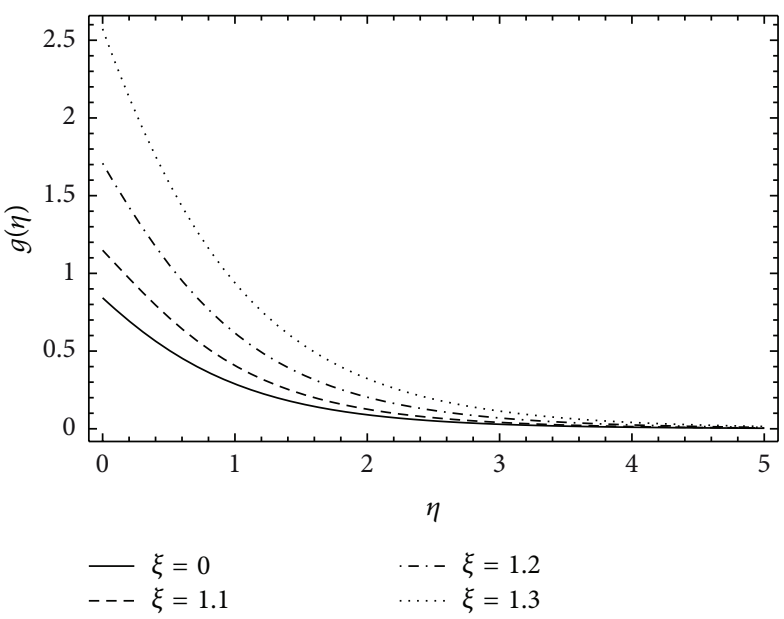

(b)

FIGURE 9: Effects of couple stress parameter $\xi$ on $g(\eta)$ (Newtonian and couple stress cases) when $\lambda=0.2$. (a) for two-dimensional when $\hbar=0.3$ and (b) for axisymmetric flow when $\hbar=0.3$.

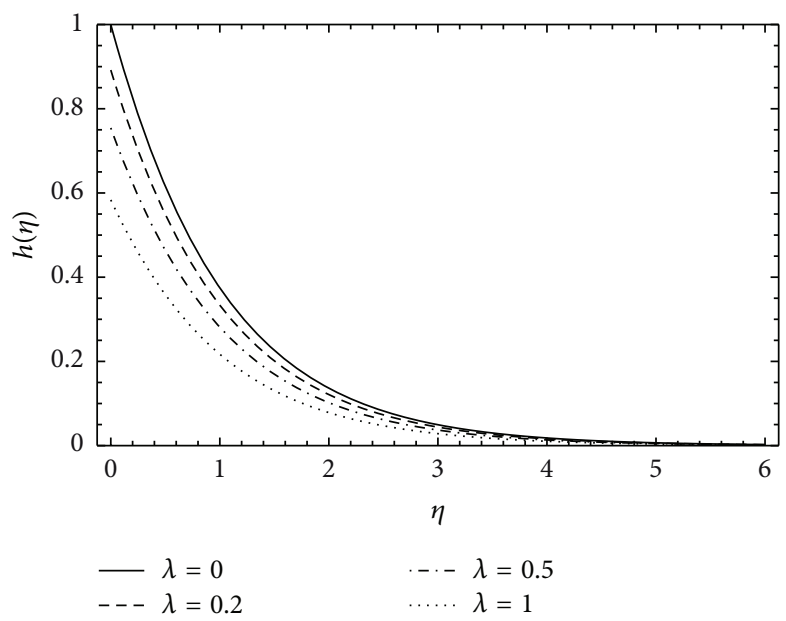

Figure 10: Effect of slip factor $\lambda$ on $h(\eta)$ (slip and no-slip cases) when $\hbar=-0.4, \xi=0.3$. 


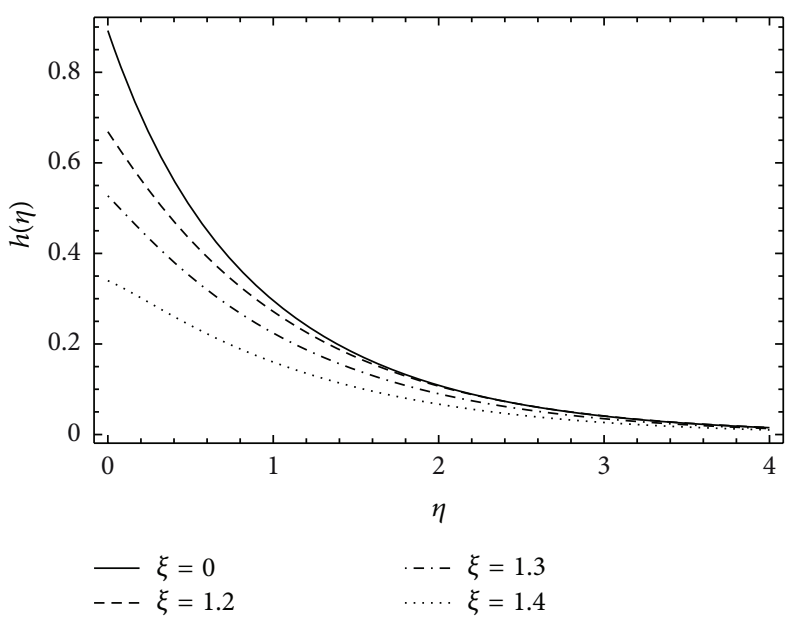

FIGURE 11: Effect of couple stress parameter $\xi$ on $h(\eta)$ (Newtonian and couple stress cases) when $\hbar=0.2, \lambda=0.2$.

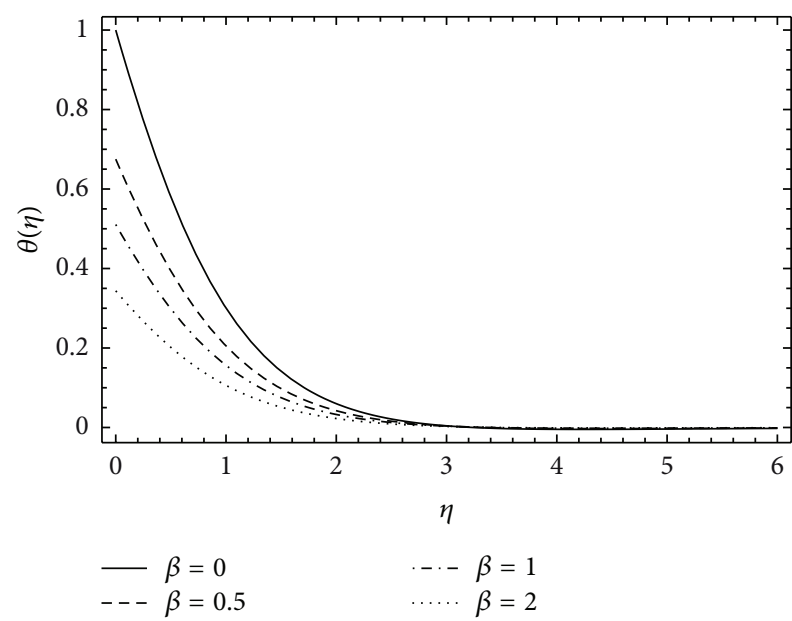

(a)

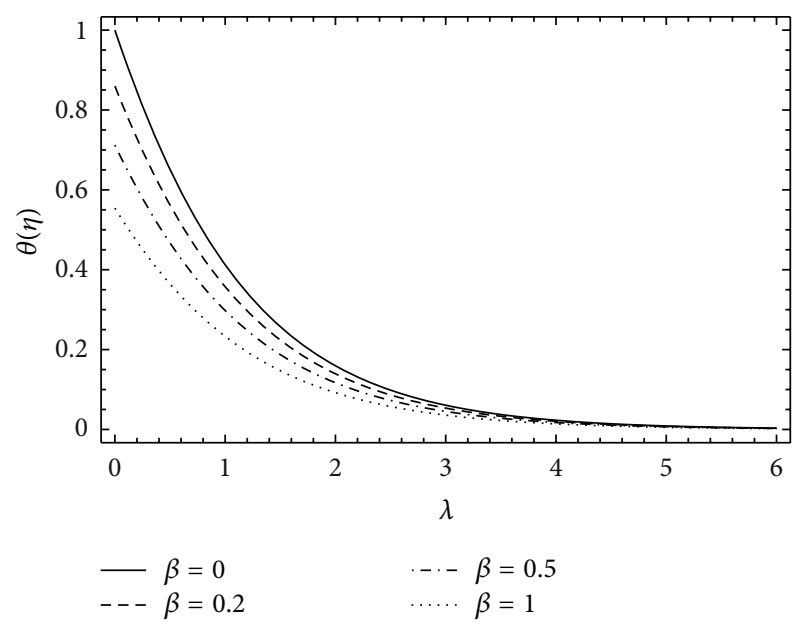

(b)

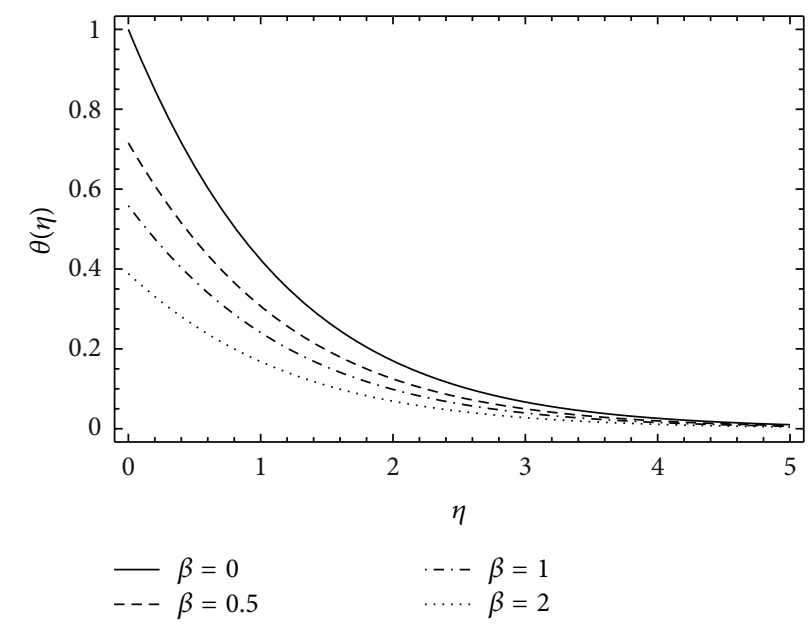

(c)

Figure 12: Temperature profile $\theta(\eta)$ of two-dimensional flow for various values of thermal slip $\beta$ (slip and no-slip cases) when $\hbar=-0.5$, $\xi=0.5$. (a) for $\operatorname{Pr}=7$. (b) for $\operatorname{Pr}=0.7$. (c) for $\operatorname{Pr}=0.07$. 


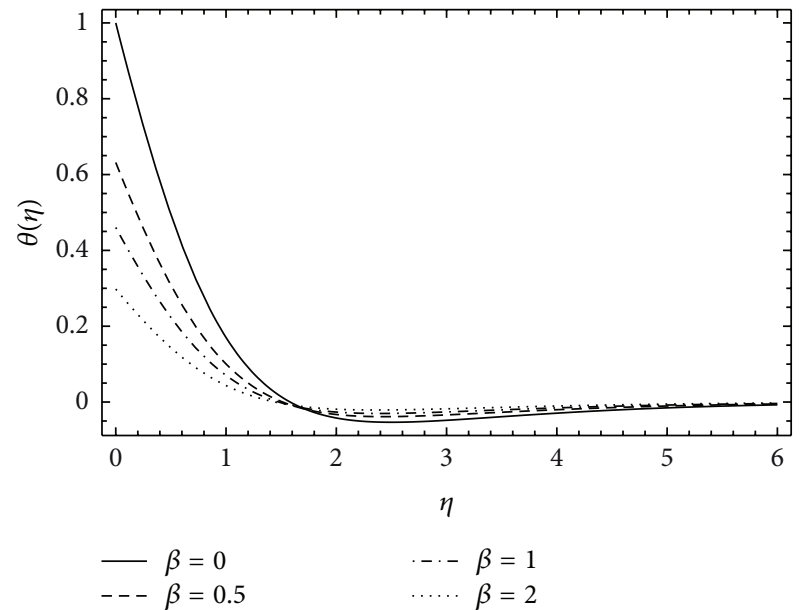

(a)

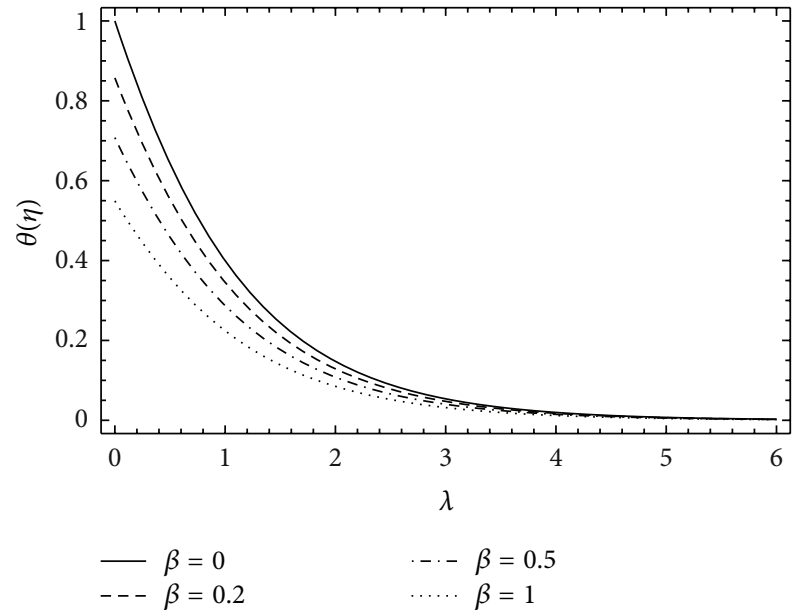

(b)

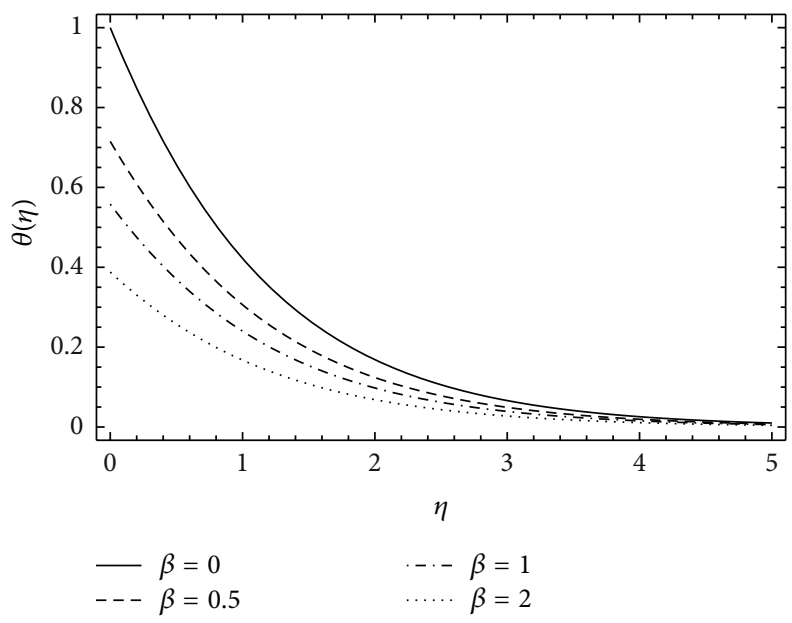

(c)

FIgURE 13: Temperature profile $\theta(\eta)$ of axisymmetric flow for various values of thermal slip $\beta$ (slip and no-slip cases) when $\hbar=-0.5, \xi=0.5$. (a) for $\operatorname{Pr}=7$. (b) for $\operatorname{Pr}=0.7$. (c) for $\operatorname{Pr}=0.07$.

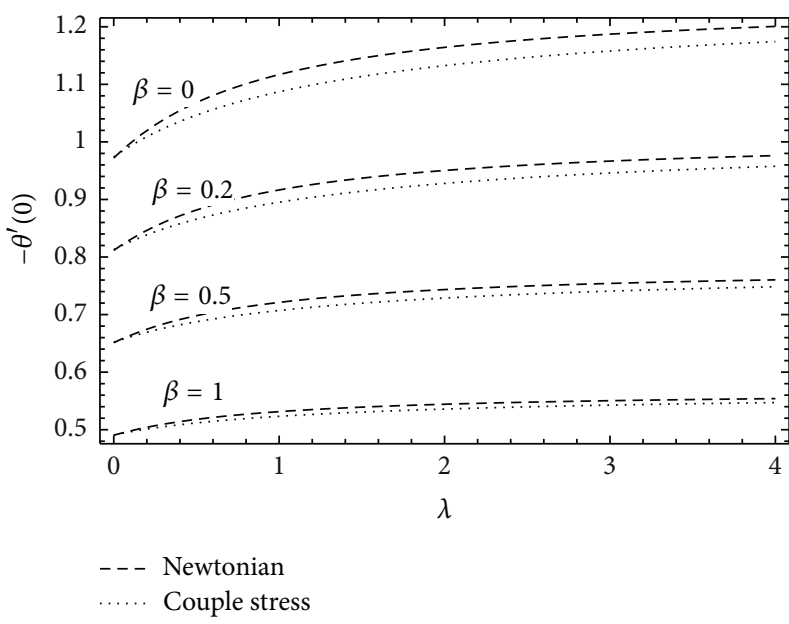

(a)

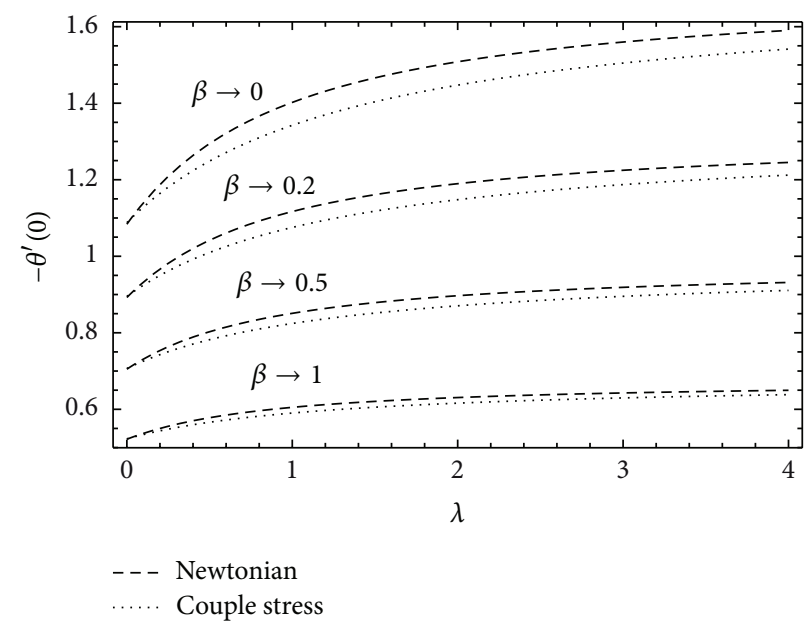

(b)

Figure 14: Profile of temperature gradient at the plate $-\theta^{\prime}(0)$ against $\lambda$ when $\operatorname{Pr}=7, \xi=0.3, \hbar=0.5$. (a) for two-dimensional. (b) for axisymmetric. 


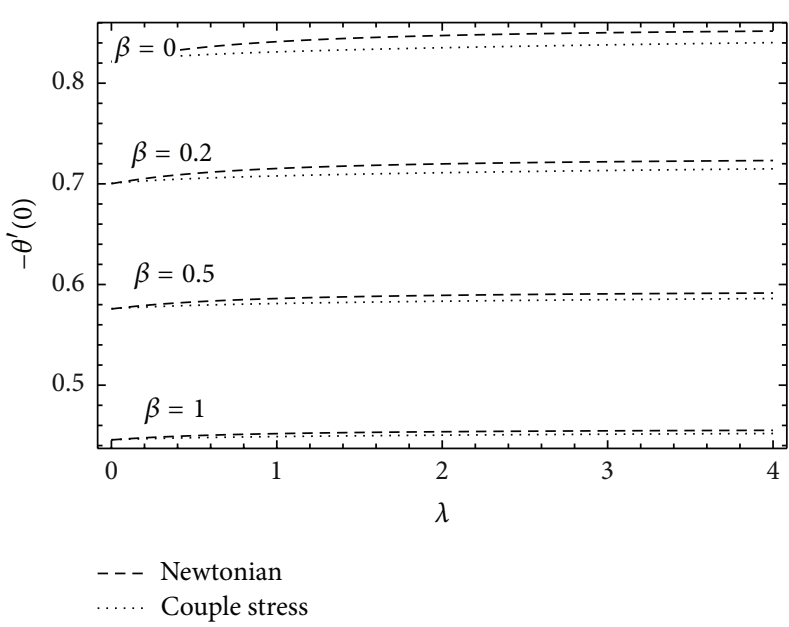

(a)

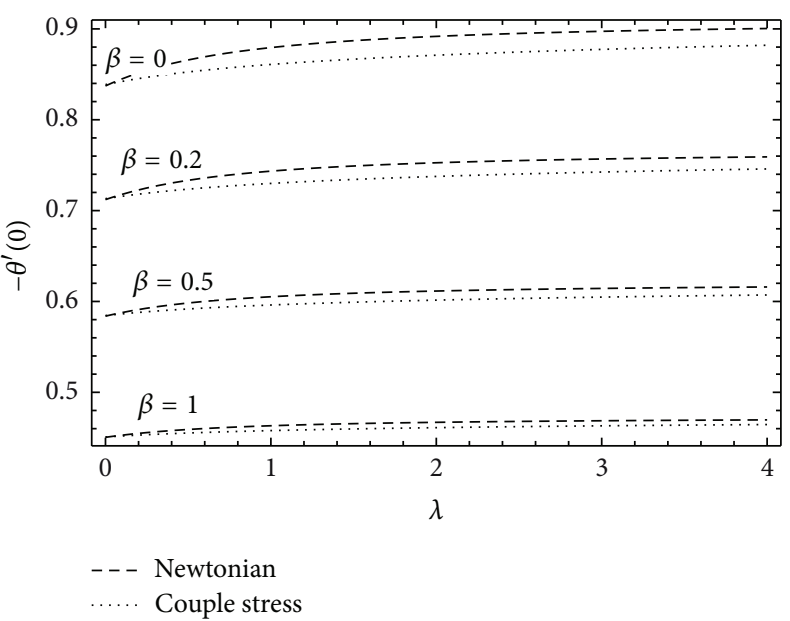

(b)

Figure 15: Profile of temperature gradient at the plate $-\theta^{\prime}(0)$ against $\lambda$ when $\operatorname{Pr}=0.7, \xi=0.3, \hbar=0.5$. (a) for two-dimensional. (b) for axisymmetric.

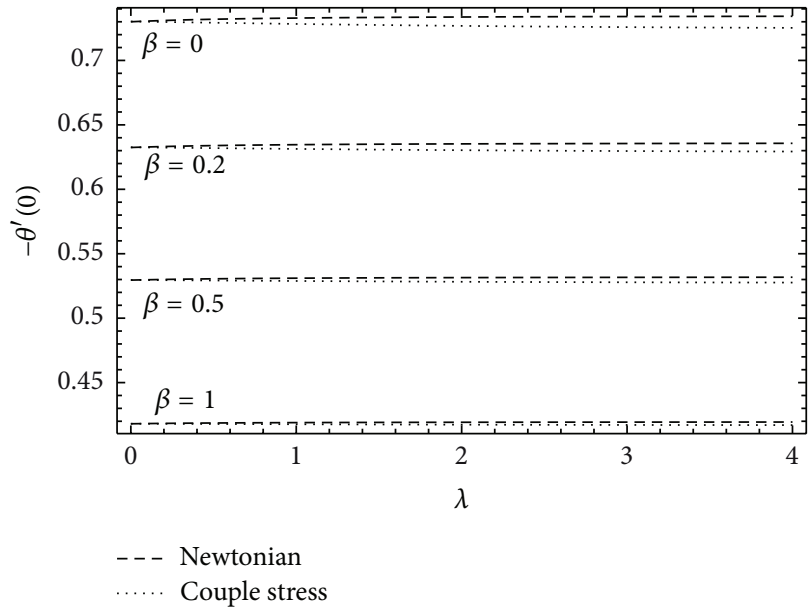

(a)

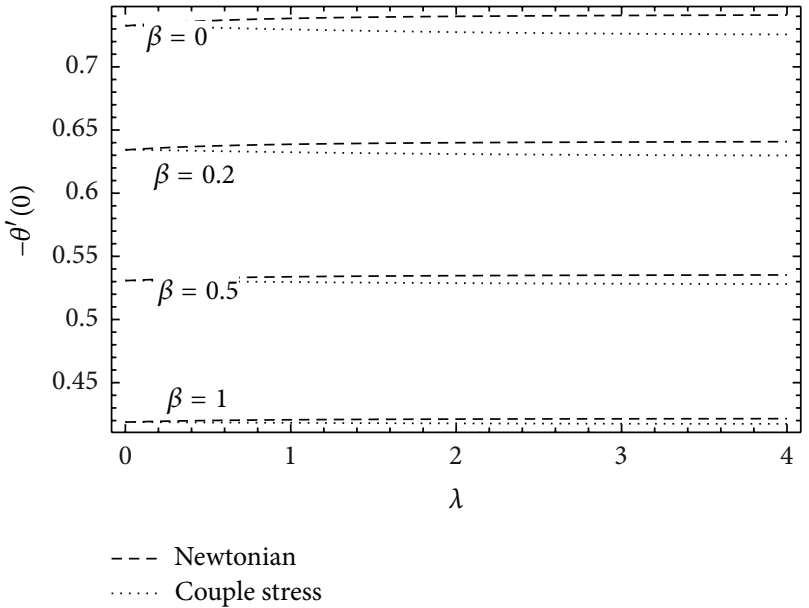

(b)

Figure 16: Profile of temperature gradient at the plate $-\theta^{\prime}(0)$ against $\beta$ when $\operatorname{Pr}=0.07, \xi=0.3, \hbar=0.5$. (a) for two-dimensional (b) for axisymmetric.

Figures 12(a), 12(b), and 12(c) for two-dimensional flow and Figures 13(a), 13(b), and 13(c) for axisymmetric flow presented the temperature profiles for various values of thermal slip $\beta$. It has been analyzed that the increment in the thermal slip $\beta$ tends to decrease the temperature of the couple stress fluid. From the definition of Prandtl number, it is quite obvious that a large Prandtl number has a lower thermal diffusivity; therefore, an increase in Pr tends to decrease the temperature.

The consequences of an increase in Prandtl number Pr and thermal slip factor $\beta$ on the heat transfer are visualized and compared with the viscous fluid considered by Wang [1] in Figures 14, 15, and 16. It is seen that the heat transfer is increasing function of $\operatorname{Pr}$ and $\lambda$ and decreasing function of $\beta$. It has also been observed that the presence of couple stresses decreases the heat transfer as compared to Newtonian fluid.
The heat transfer coefficient for axisymmetric flow is smaller as compared with large value of $-\theta^{\prime}(0)$ for two-dimensional flow.

\section{Conclusions}

The study has been done to explore the influence of couple stresses with partial slip condition on the flow pattern of couple stress fluid with heat transfer.

(i) The trends for the physical parameters of skin frictions, $C_{f}, C_{g}$, and $C_{h}$ for two-dimensional and axisymmetric flows are decreasing function of slip factor.

(ii) The velocity functions $f^{\prime}$ and $f$ are increasing functions of slip factor $\lambda(0 \leq \lambda<\infty)$. 
(iii) The axial velocity $g$ and translational velocity $h$ are decreasing functions of slip factor $\lambda$.

(iv) Couple stress parameter $\xi$ has the negative impact on velocity functions $f^{\prime}, f$ on both two-dimensional and axisymmetric flows.

(v) The axial velocity $g$ increases in two-dimensional flow and decreases in axisymmetric flow for the increasing values of couple stress parameter $\xi$.

(vi) The velocity function $h$ found no support from the couple stresses in the fluid for the flow in translational direction in both type of flows.

(vii) The temperature of the couple stress fluid is the decreasing function of thermal slip $\beta$.

(viii) Prandtl number has the positive effect on the heat transfer of the fluid.

\section{Notations}

$\begin{array}{ll}(x, y, z): & \text { Space coordinates } \\ (u, v, w): & \text { Velocity components } \\ v: & \text { Kinematic viscosity } \\ \rho: & \text { Density } \\ k: & \text { Thermal conductivity } \\ c_{p}: & \text { Specific heat } \\ T: & \text { Temperature of fluid } \\ \gamma: & \text { Couple stress parameter } \\ N, S: & \text { Slip constants } \\ \operatorname{Pr}: & \text { Prandtl number } \\ \xi: & \text { Dimensionless couple stress parameter } \\ a, U, V: & \text { Dimensional rate constant } \\ \lambda: & \text { Nondimensional slip factor } \\ \beta: & \text { Thermal slip factor } \\ \operatorname{Re}: & \text { Reynolds number } \\ C_{f}, C_{g}, C_{h}: & \text { Skin friction coefficients. }\end{array}$

\section{Conflict of Interests}

The authors declare that they have no conflict of interests.

\section{Authors' Contribution}

The work including proofreading was done by all the authors.

\section{Acknowledgment}

The author Najeeb Alam Khan is thankful and grateful to the Dean of Faculty of Sciences, University of Karachi, Karachi, Pakistan, for supporting and facilitating this research work.

\section{References}

[1] C. Y. Wang, "Stagnation slip flow and heat transfer on a moving plate," Chemical Engineering Science, vol. 61, no. 23, pp. 76687672, 2006.

[2] P. D. Ariel, "Axisymmetric flow due to a stretching sheet with partial slip," Computers and Mathematics with Applications, vol. 54, no. 7-8, pp. 1169-1183, 2007.
[3] S. Nadeem, T. Hayat, S. Abbasbandy, and M. Ali, "Effects of partial slip on a fourth-grade fluid with variable viscosity: an analytic solution," Nonlinear Analysis: Real World Applications, vol. 11, no. 2, pp. 856-868, 2010.

[4] B. Sahoo, "Flow and heat transfer of a non-Newtonian fluid past a stretching sheet with partial slip," Communications in Nonlinear Science and Numerical Simulation, vol. 15, no. 3, pp. 602-615, 2010.

[5] M. Jamil and N. A. Khan, "Slip effects on fractional viscoelastic fluids," International Journal of Differential Equations, vol. 2011, Article ID 193813, 19 pages, 2011.

[6] V. Kumaran and I. Pop, "Nearly parallel blasius flow with slip," Communications in Nonlinear Science and Numerical Simulation, vol. 16, no. 12, pp. 4619-4624, 2011.

[7] V. K. Stokes, "Couple stresses in fluids," Physics of Fluids, vol. 9, no. 9, pp. 1709-1715, 1966.

[8] S. Nadeem and S. Akram, "Peristaltic flow of a couple stress fluid under the effect of induced magnetic field in an asymmetric channel," Archive of Applied Mechanics, vol. 81, no. 1, pp. 97-109, 2011.

[9] N. A. Khan, A. Mahmood, and A. Ara, "Approximate solution of couple stress fluid with expanding or contracting porous channel," Engineering Computations, vol. 30, no. 3, pp. 399-408, 2013.

[10] J. V. Ramana Murthy and G. Nagaraju, "Flow of a couple stress fluid generated by a circular cylinder subjected to longitudinal and torsional oscillations," Contemporary Engineering Sciences, vol. 2, no. 10, pp. 451-461, 2009.

[11] T. Hayat, M. Mustafa, Z. Iqbal, and A. Alsaedi, "Stagnationpoint flow of couple stress fluid with melting heat transfer," Applied Mathematics and Mechanics, vol. 34, no. 2, pp. 167-176, 2013.

[12] M. Devakar and T. K. V. Iyengar, "Run up flow of a couple stress fluid between parallel plates," Nonlinear Analysis: Modelling and Control, vol. 15, no. 1, pp. 29-37, 2010.

[13] S. Liang and D. J. Jeffrey, "Comparison of homotopy analysis method and homotopy perturbation method through an evolution equation," Communications in Nonlinear Science and Numerical Simulation, vol. 14, no. 12, pp. 4057-4064, 2009.

[14] M. Sajid, T. Hayat, and S. Asghar, "Comparison between the HAM and HPM solutions of thin film flows of non-Newtonian fluids on a moving belt," Nonlinear Dynamics, vol. 50, no. 1-2, pp. 27-35, 2007.

[15] S. Abbasbandy, "The application of homotopy analysis method to nonlinear equations arising in heat transfer," Physics Letters A, vol. 360, no. 1, pp. 109-113, 2006.

[16] F. M. Allan and M. I. Syam, "On the analytic solutions of the nonhomogeneous Blasius problem," Journal of Computational and Applied Mathematics, vol. 182, no. 2, pp. 362-371, 2005.

[17] T. Hayat and T. Javed, "On analytic solution for generalized three-dimensional MHD flow over a porous stretching sheet," Physics Letters A, vol. 370, no. 3-4, pp. 243-250, 2007.

[18] T. Hayat and M. Sajid, "Analytic solution for axisymmetric flow and heat transfer of a second grade fluid past a stretching sheet," International Journal of Heat and Mass Transfer, vol. 50, no. 1-2, pp. 75-84, 2007.

[19] S. Abbasbandy, "Homotopy analysis method for the Kawahara equation," Nonlinear Analysis: Real World Applications, vol. 11, no. 1, pp. 307-312, 2010.

[20] N. A. Khan, M. Jamil, and K. A. Nadeem, "Effects of slip factors on unsteady stagnation point flow and heat transfer towards a stretching sheet: an analytical study," Heat Transfer Research, vol. 43, no. 8, pp. 779-794, 2012. 

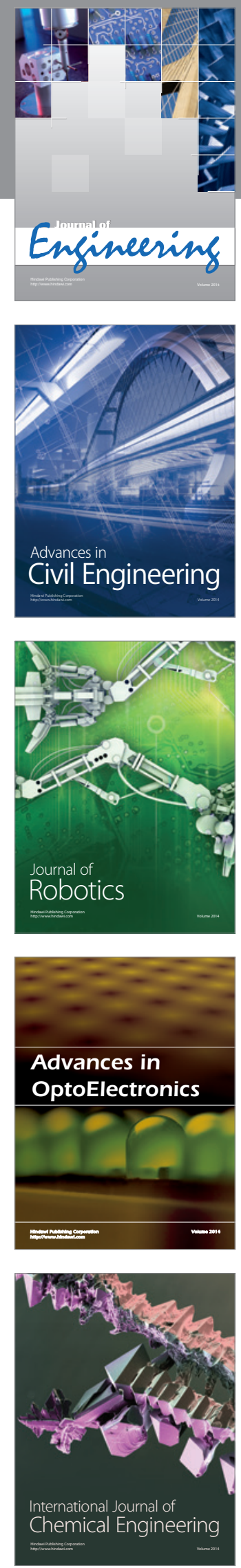

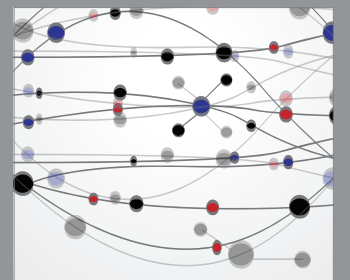

The Scientific World Journal
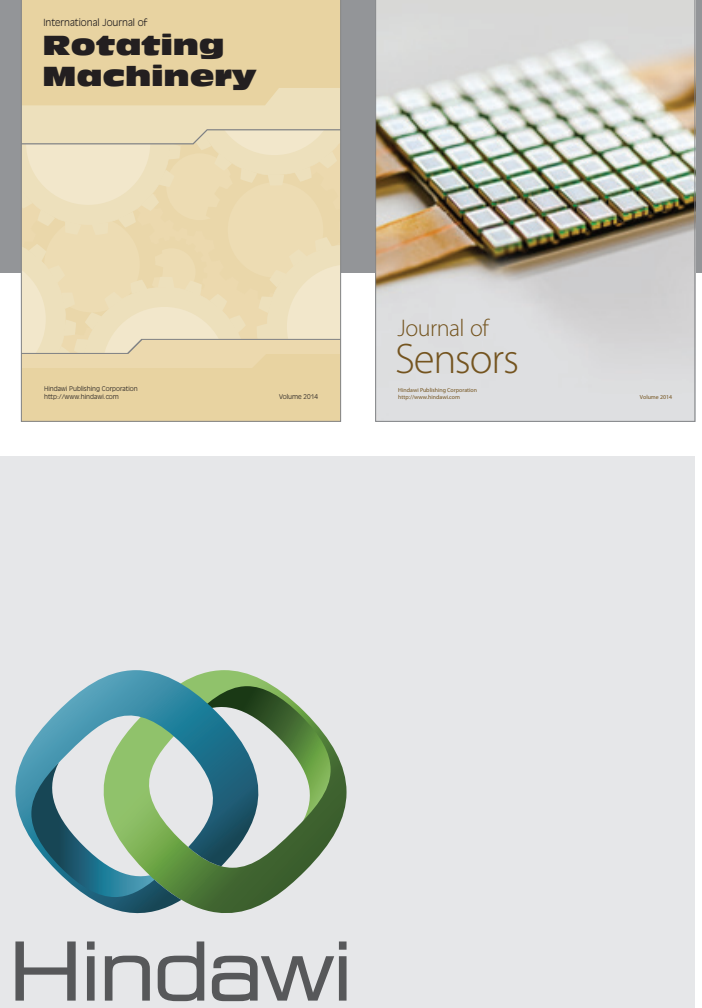

Submit your manuscripts at http://www.hindawi.com
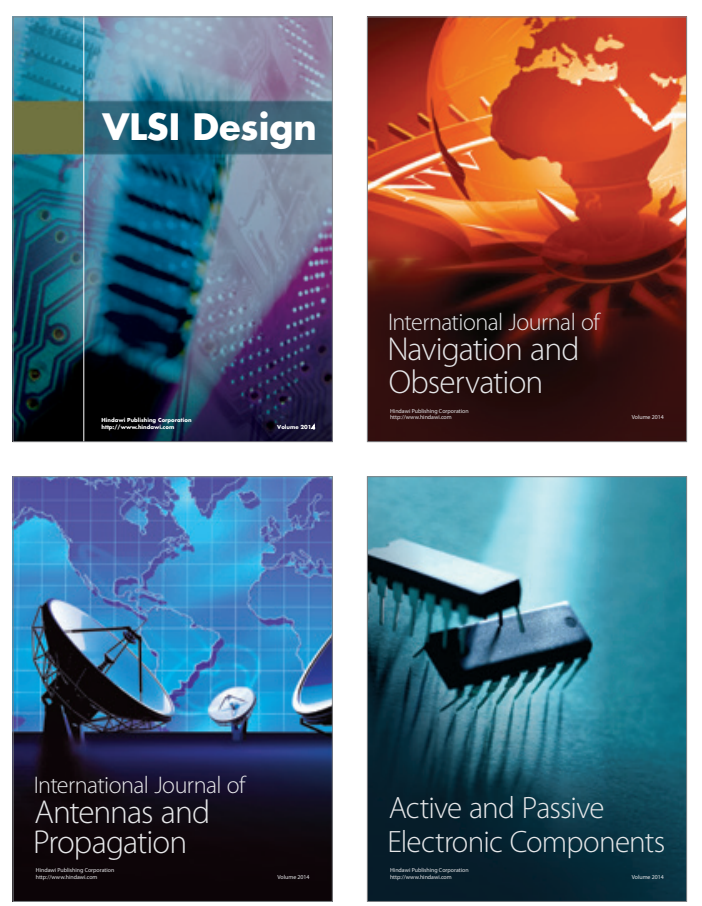
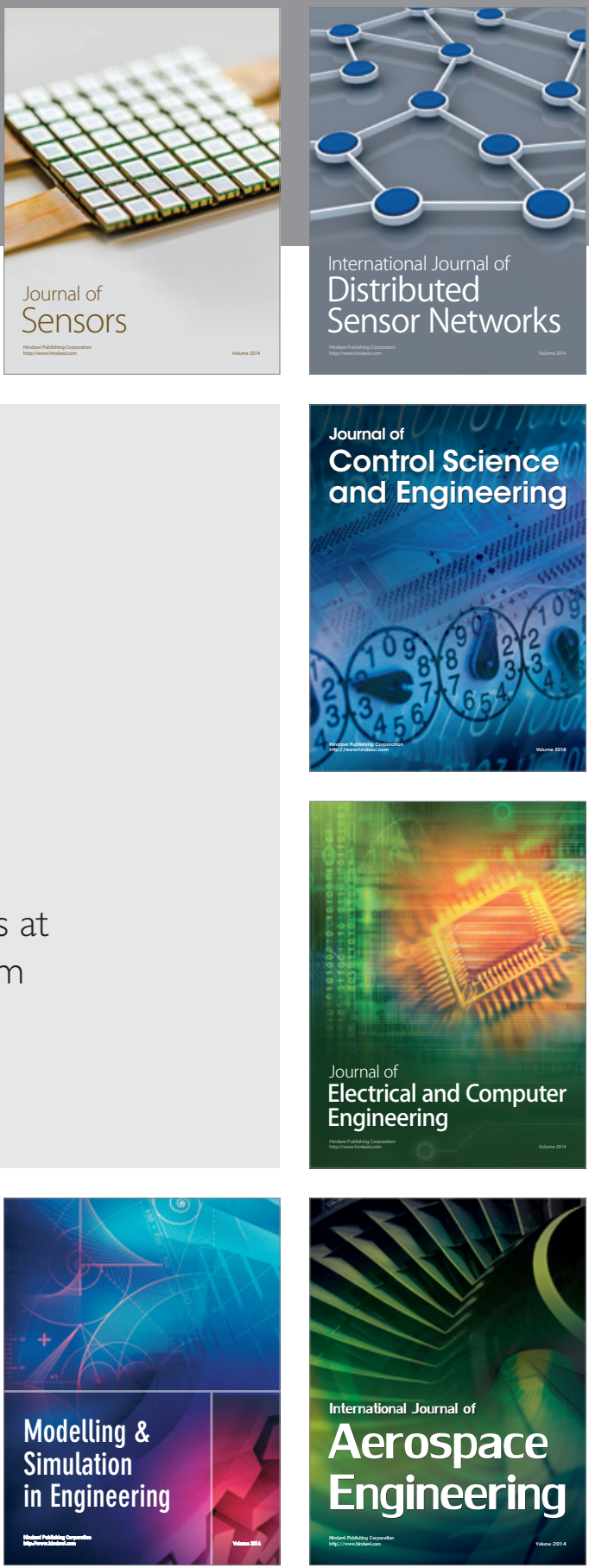

Journal of

Control Science

and Engineering
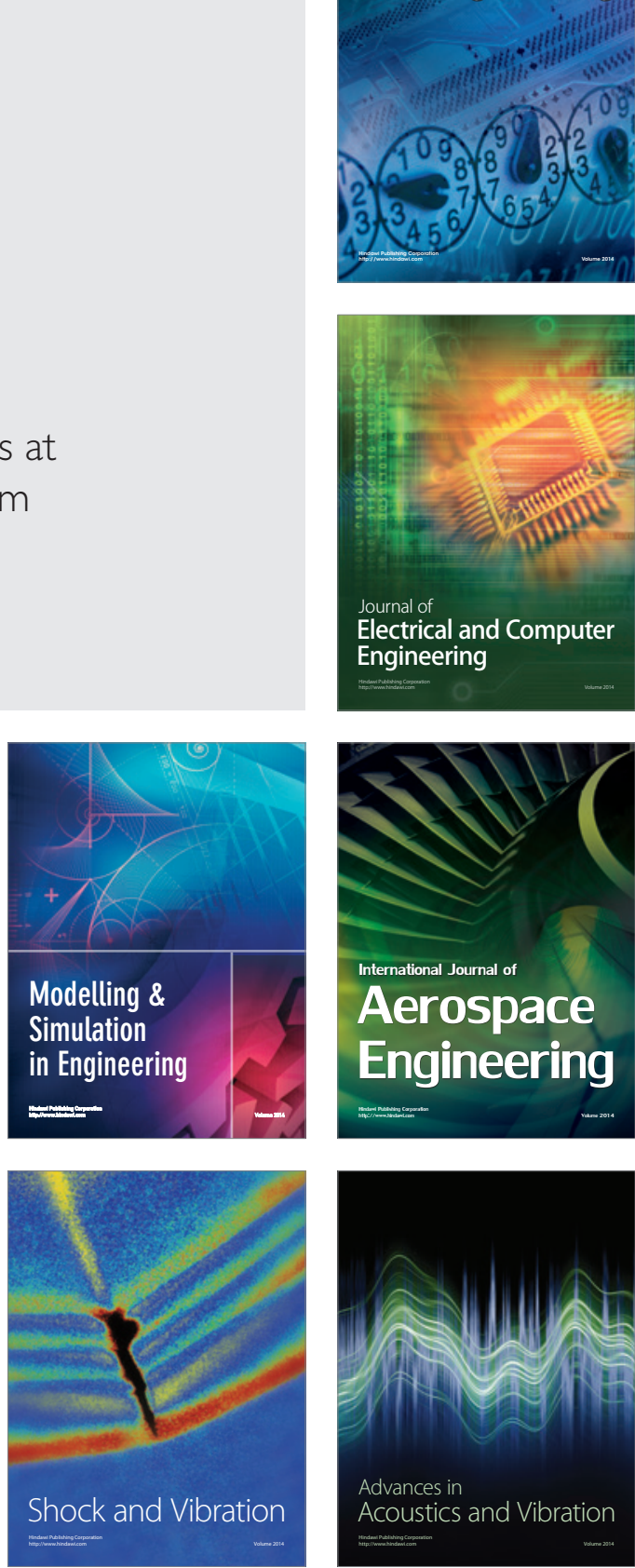